J. Alberto Neder ${ }^{1,2}$, Roberta P. Ramos ${ }^{1,3}$, Jaquelina S. Ota-Arakaki ${ }^{1,3}$, Eloara M.V. Ferreira ${ }^{1,3}$, Daniel M. Hirai ${ }^{1,2}$, Priscila A. Sperandio ${ }^{1}$, Maria Clara N. Alencar ${ }^{1}$, Flavio F. Arbex ${ }^{1}$, Danilo C. Berton ${ }^{4}$, Christine D'Arsigny ${ }^{2}$ and Denis E. O'Donnell ${ }^{2}$

${ }^{1}$ Pulmonary Function and Clinical Exercise Physiology Unit, Respiratory Division, Dept of Medicine, School of Medicine, Federal University of São Paulo, São Paulo, Brazil. ${ }^{2}$ Laboratory of Clinical Exercise Physiology and Respiratory Investigation Unit, Division of Respiratory and Critical Care Medicine, Dept of Medicine, Queen's University, Kingston, ON, Canada. ${ }^{3}$ Pulmonary Vascular Group, Respiratory Division, Dept of Medicine, School of Medicine, Federal University of São Paulo, São Paulo, Brazil. ${ }^{4}$ Respiratory Division, Dept of Medicine, Federal University of Rio Grande do Sul, Porto Alegre, Brazil.

Correspondence: J. Alberto Neder, Division of Respiratory and Critical Care Medicine, Queen's University and Kingston General Hospital, Richardson House, 102 Stuart Street, Kingston, ON K7L 2V6, Canada. E-mail: nederalb@gmail.com

Received: Nov 212015 | Accepted after revision: Feb 282016 | First published online: April 132016

Conflict of interest: None declared.

\title{
References
}

Mélot C, Naeije R. Pulmonary vascular diseases. Compr Physiol 2011; 1: 593-619.

2 Neder JA, Ramos RP, Ota-Arakaki JS, et al. Exercise intolerance in pulmonary arterial hypertension. The role of cardiopulmonary exercise testing. Ann Am Thorac Soc 2015; 12: 604-612.

3 McCabe C, Deboeck G, Harvey I, et al. Inefficient exercise gas exchange identifies pulmonary hypertension in chronic thromboembolic obstruction following pulmonary embolism. Thromb Res 2013; 132: 659-665.

4 Claessen G, La Gerche A, Wielandts J-Y, et al. Exercise pathophysiology and sildenafil effects in chronic thromboembolic pulmonary hypertension. Heart Br Card Soc 2015; 101: 637-644.

5 van der Plas MN, Reesink HJ, Roos CM, et al. Pulmonary endarterectomy improves dyspnea by the relief of dead space ventilation. Ann Thorac Surg 2010; 89: 347-352.

6 Hansen JE, Ulubay G, Chow BF, et al. Mixed-expired and end-tidal $\mathrm{CO}_{2}$ distinguish between ventilation and perfusion defects during exercise testing in patients with lung and heart diseases. Chest 2007; 132: 977-983.

7 Yasunobu Y, Oudiz RJ, Sun X-G, et al. End-tidal $\mathrm{PCO}_{2}$ abnormality and exercise limitation in patients with primary pulmonary hypertension. Chest 2005; 127: 1637-1646.

8 Scheidl SJ, Englisch C, Kovacs G, et al. Diagnosis of CTEPH versus IPAH using capillary to end-tidal carbon dioxide gradients. Eur Respir J 2012; 39: 119-124.

9 Dantzker DR, Bower JS. Mechanisms of gas exchange abnormality in patients with chronic obliterative pulmonary vascular disease. J Clin Invest 1979; 64: 1050-1055.

10 Delcroix M, Vonk Noordegraaf A, Fadel E, et al. Vascular and right ventricular remodelling in chronic thromboembolic pulmonary hypertension. Eur Respir J 2013; 41: 224-232.

11 Naeije R, van de Borne P. Clinical relevance of autonomic nervous system disturbances in pulmonary arterial hypertension. Eur Respir J 2009; 34: 792-794.

12 Theodore J, Robin ED, Morris AJ, et al. Augmented ventilatory response to exercise in pulmonary hypertension. Chest 1986; 89: 39-44.

13 Zhai Z, Murphy K, Tighe H, et al. Differences in ventilatory inefficiency between pulmonary arterial hypertension and chronic thromboembolic pulmonary hypertension. Chest 2011; 140: 1284-1291.

14 Whipp BJ. The bioenergetic and gas exchange basis of exercise testing. Clin Chest Med 1994; 15: 173-192.

15 Ward SA, Whipp BJ, Koyal S, et al. Influence of body $\mathrm{CO}_{2}$ stores on ventilatory dynamics during exercise. J Appl Physiol 1983; 55: 742-749.

\section{Diagnostic concordance of different criteria for exercise pulmonary hypertension in subjects with normal resting pulmonary artery pressure}

To the Editor:

Pulmonary hypertension is defined by a resting mean pulmonary artery pressure (mPAP) $\geqslant 25 \mathrm{mmHg}$ [1]. Despite a better understanding of the biology of pulmonary hypertension and new therapeutic advances, pulmonary hypertension remains diagnosed late in its natural history and is largely a non-curable condition [2]. Recently, there has been renewed interest in stress-testing of the pulmonary circulation since the early stages of pulmonary vascular disease (PVD) or left heart disease (LHD) can be associated with normal resting mPAP but an abnormal haemodynamic response that is unmasked by exercise [3-5]. 
Although no consensus definition currently exists for the diagnosis of exercise pulmonary hypertension, any criteria for exercise pulmonary hypertension should incorporate an assessment of mPAP-cardiac output (mPAP-CO) relationship from a physiological perspective, since mPAP is a flow-dependent variable. Current evidence suggests that in health, $\mathrm{mPAP}$ should not exceed $30 \mathrm{mmHg}$ at a cardiac output of $<10 \mathrm{~L} \cdot \mathrm{min}^{-1}$ [3]. However, multiple methods of mPAP-CO assessment have been proposed in the literature for exercise stress-testing of the pulmonary circulation, and it is unknown whether these differing methods necessarily produce concordant results.

A recent clinical study by our group has favoured the criteria for exercise pulmonary hypertension using the combined haemodynamic parameters of peak mPAP (mPAPmax) $>30 \mathrm{mmHg}$ and peak total pulmonary resistance (TPRmax) $>3 \mathrm{WU}$ during exercise [5]. TPRmax uses a single-point mPAP-CO ratio at peak exercise. This combined criterion has been shown to significantly reduce the number of healthy controls who are misclassified as having exercise pulmonary hypertension compared with the previous definition of exercise pulmonary hypertension ( $\mathrm{mPAP}>30 \mathrm{mmHg}$ ) [6]. An alternative method of mPAP-CO assessment for the diagnosis of exercise pulmonary hypertension uses the slope of multipoint mPAP-CO relationships taken at multiple levels of exercise (generally 4-5 data points), and an mPAP-CO slope $>3 \mathrm{WU}$ is used to define exercise pulmonary hypertension [4]. Finally, a method that uses a two-point measurement of the mPAP-CO slope from resting and peak exercise haemodynamics has also been proposed [7, 8]. Although these different methods of assessing the mPAP-CO relationship appear "similar", they are not synonymous when one considers carefully the behaviour of the $\mathrm{MPAP}-\mathrm{CO}$ relationship during exercise. The aim of our study was to evaluate the diagnostic concordance of the different methods that have been proposed for the assessment of the mPAP-CO relationship for the diagnosis of exercise pulmonary hypertension.

A total of 169 subjects ( $\mathrm{n}=68$ controls; $\mathrm{n}=49$ PVD; $\mathrm{n}=52$ LHD) with normal resting mPAP $\leqslant 20 \mathrm{mmHg}$ underwent exercise haemodynamic evaluation with lower limb cycle ergometry. The detailed exercise protocol has been reported previously [9]. This retrospective study was approved by the ethics board of the Université Paris-Sud (approval no. 9708) and informed consent was obtained from all patients.

Patients were defined as displaying exercise pulmonary hypertension according to each of the following three diagnostic criteria: 1) exercise mPAPmax $>30 \mathrm{mmHg}$ plus exercise TPRmax $>3 \mathrm{WU}$ (mPAPmax $>30 \mathrm{mmHg}$ $+\mathrm{TPR} \max >3 \mathrm{WU}$ ); 2) linearised slope of multiple mPAP-CO relationships $>3 \mathrm{WU}$ (mPAP-COslope $>3 \mathrm{WU}$ ); and 3) the ratio of mPAPmax minus resting mPAP over peak cardiac output minus resting cardiac output $>3 \mathrm{WU}(\triangle \mathrm{mPAP} / \triangle \mathrm{CO}>3 \mathrm{WU})$. Diagnostic concordance was then assessed based on the percentage of cases with concordant classification and the kappa statistic. Statistical analyses were performed using SPSS v22 (IBM, Chicago, IL, USA).

Detailed demographics of the study population, including resting and exercise haemodynamic results, have been reported elsewhere [5]. The average number of mPAP-CO points available from rest to peak exercise was (5.4 \pm 1.7$)$. For the mPAP-COslope method, the median $\mathrm{R}^{2}$ of the linear regression fit of mPAP-CO points was 0.86 (range $0.32-0.99$ ).

All three criteria were associated with high diagnostic accuracy for the discrimination of controls from patients with PVD and LHD, with respective area under the curve (AUC) on ROC analysis as follows: mPAPmax + TPRmax $=0.99, \mathrm{mPAP}-\mathrm{CO}$ slope $=0.94$, and $\Delta \mathrm{mPAP} / \Delta \mathrm{CO}=0.96$. Table 1 summarises the respective sensitivies and specificities of the three different haemodynamic criteria for the discriminaton of controls versus patients with LHD and PVD.

When the three different criteria with their respective cut-off values were used to classify patients into the presence or absence of exercise pulmonary hypertension, concordant classification was found in $80.5 \%$ of cases for mPAPmax+TPRmax versus mPAP-COslope $(\kappa=0.61) ; 85.8 \%$ for mPAP+TPRmax versus $\Delta \mathrm{mPAP} /$ $\Delta \mathrm{CO}(\kappa=0.71)$; and $84.0 \%$ for mPAP-COslope versus $\Delta \mathrm{mPAP} / \Delta \mathrm{CO}(\kappa=0.68)$.

Overall, $78 \%$ of cases were fully concordant across all three criteria. Diagnostic disagreement were very uncommon for cases with either very flat mPAP-CO response (TPRmax $<2 \mathrm{WU})(0 \%)$ or steep mPAP-CO response (TPRmax $>4 \mathrm{WU}$ ) (6\%). The value of the zero-flow pressure intercept for cases that were fully concordant for all three criteria was not significantly different compared with cases with diagnostic disagreement (median (interquartile range), $1.5(-3.4-5.6)$ versus $1.3(-8.8-6.4) \mathrm{mmHg}$; $=0.44)$.

The present study highlights that the different criteria that have been recently proposed for the diagnosis of exercise pulmonary hypertension are not interchangeable. Although all of these criteria incorporate an assessment of mPAP-CO relationship during exercise (in contrast to the old definition which contained only a pressure parameter), the differing methodologies of each criteria may result in significant disagreement for the diagnosis of exercise pulmonary hypertension.

Pulmonary pressure-flow relationship is usually well described by linear relationships over physiological flow ranges but this is only an approximation. In health, the mPAP-CO relationship when left atrial 
TABLE 1 Diagnostic performance of various criteria for discriminating controls versus patients with PVD and LHD

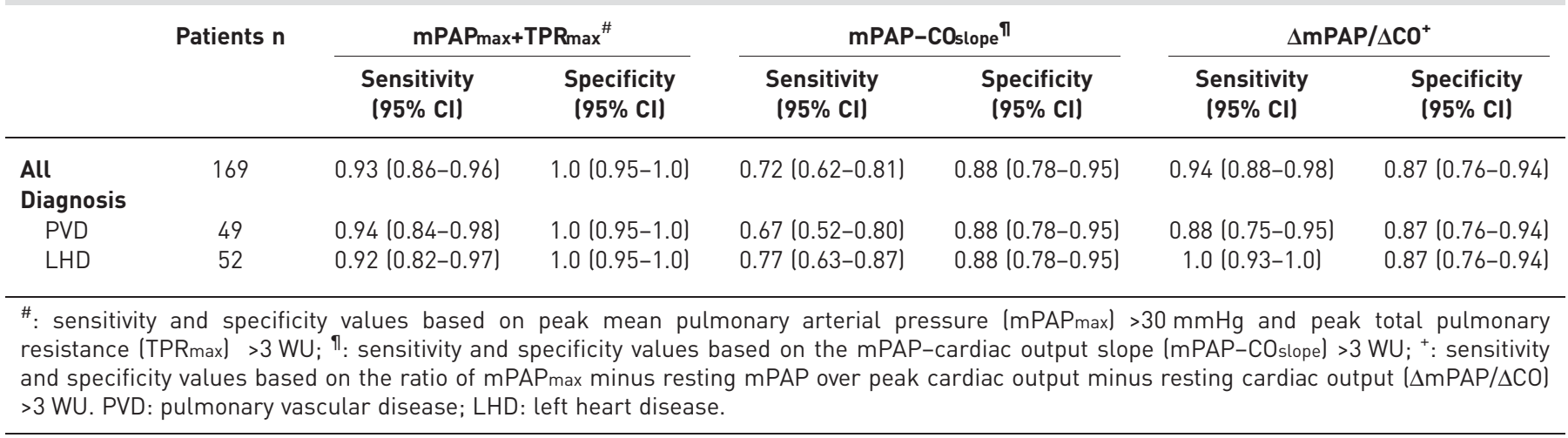

pressure is held unchanged may actually display a slight curvilinearity [10], owing to the natural distensibility of the pulmonary circulation. Other non-linear patterns of mPAP-CO relationship have also been described in disease states such as pulmonary arterial hypertension [11].

Using a simple linear model, the mPAP-CO relationship can be expressed as $y=a x+b$, where $y$ is mPAP, $x$ is $\mathrm{CO}$ and $\mathrm{b}$ is the extrapolated pressure intercept at zero flow. It can be easily appreciated that for all of the criteria to be fully consistent, the following conditions must be met: 1) the zero-flow pressure intercept must cross origin, and 2) the mPAP-CO relationship must be strictly linear over the measured flow ranges. Given that these assumptions are not always met, it is not surprising that diagnostic disagreement is present amongst the different criteria for exercise pulmonary hypertension. Furthermore, haemodynamic measurements at maximal exercise may be more sensitive at detecting late-exercise surge in pulmonary artery pressure induced by low mixed venous oxygen content and sympathetic nervous system activation [12], or excessive late-exercise rise in left atrial pressure. Accordingly, both the mPAPmax+TPRmax and the $\triangle \mathrm{PAP} / \triangle \mathrm{CO}$ methods place greater emphasis on the single measurement obtained at peak exercise, whereas the mPAP-COslope method characterises mPAP-CO response across all exercise stages. The different criteria also have practical implications for exercise haemodynamic testing since linear regression analysis of multipoint measurements at many different workloads (mPAP-COslope) is more complex, in comparison to the more simple approach in which exercise pulmonary hypertension is diagnosed whenever mPAP exceeds $30 \mathrm{mmHg}$ at an equivalent cardiac output of $<10 \mathrm{~L} \cdot \mathrm{min}^{-1}$ (mPAPmax+TPRmax). However, it must be acknowledged that this is simply a practical observation and detailed mPAP-CO plots (with $>4-5$ points) afford a more detailed description of vascular resistance during exercise.

For the majority of healthy subjects who have very flat pressure-flow relationships, all three criteria will allow correct classification as normal response. Conversely, for many patients with exercise pulmonary hypertension and very steep pressure-flow response, they will also exceed the threshold set by all three criteria. However, there will remain a significant number of subjects who will have discrepant classification, as demonstrated by our results. Imprecise pressure and cardiac output measurements in clinical practice may also contribute to our findings. Limitations of the current study include a small number of subjects above the age of 70 years, patients with a diagnosis of PVD were over-represented by chronic thromboembolic disease, and the majority of subjects were women.

In conclusion, our study demonstrates that the different methods used to define mPAP-CO relationship for the diagnosis of exercise pulmonary hypertension suffer from lack of diagnostic concordance in a substantial number of cases. This is particularly relevant for patients who have exercise mPAP-CO response close to the current threshold of $3 \mathrm{WU}$ proposed in the current literature. Significant advancement has already been made to incorporate the assessment of flow and not merely pressure for the diagnosis of exercise pulmonary hypertension. The scientific community must now come to an agreement on a practical and robust definition of exercise pulmonary hypertension in order for progress to occur in this field.

@ERSpublications

Methodology of pressure-flow assessment needs to be standardized for the diagnosis of exercise pulmonary hypertension http://ow.ly/YMgZw

Laurent Godinas ${ }^{1,2,3,4}$, Edmund M. Lau ${ }^{1,2,3,5}$, Denis Chemla ${ }^{1,3,6}$, Frédéric Lador $\odot^{7}$, Laurent Savale ${ }^{1,2,3}$, David Montani ${ }^{1,2,3}$, Xavier Jaïs ${ }^{1,2,3}$, Olivier Sitbon ${ }^{1,2,3}$, Gérald Simonneau ${ }^{1,2,3}$, Marc Humbert $\oplus^{1,2,3}$ and Philippe Hervé (1,3,8 $^{2}$ 
${ }^{1}$ Faculté de Médecine, Université Paris-Sud, Université Paris-Saclay, Le Kremlin-Bicêtre, France. ${ }^{2}$ Service de Pneumologie, AP-HP, Le Kremlin-Bicetre, France. ${ }^{3}$ INSERM UMR_S999, Hôpital Marie Lannelongue, Le Plessis Robinson, France. ${ }^{4}$ Service de Pneumologie, CHU UcL Namur, Yvoir, Belgium. ${ }^{5}$ Sydney Medical School, University of Sydney, Camperdown, Australia. ${ }^{6}$ Département de Physiologie, AP-HP, Le Kremlin-Bicetre, France. ${ }^{7}$ Service de Pneumologie - Programme Hypertension Pulmonaire, Hôpitaux Universitaires de Genève, Geneva, Switzerland. ${ }^{8}$ Département de Chirurgie Thoracique, Vasculaire et de Transplantation Pulmonaire, Hôpital Marie Lannelongue, Le Plessis Robinson, France.

Correspondence: Edmund M. Lau, Department of Respiratory and Sleep Medicine, Royal Prince Alfred Hospital, University of Sydney, Missenden Rd, Camperdown NSW 2050, Australia. E-mail: edmund.lau@sydney.edu.au

Received: Oct 102015 | Accepted after revision: Feb 182016 | First published online: March 302016

Support statement: Funding for this manuscript was provided by Fondation Mont-Godinne.

Conflict of interest: Disclosures can be found alongside this article at erj.ersjournals.com

\section{References}

1 Hoeper MM, Bogaard HJ, Condliffe R, et al. Definitions and diagnosis of pulmonary hypertension. J Am Coll Cardiol 2013; 62: Suppl. 25, D42-D50.

2 Lau EMT, Humbert M, Celermajer DS. Early detection of pulmonary arterial hypertension. Nat Rev Cardiol 2014; 12: 143-155.

3 Naeije R, Vanderpool R, Dhakal BP, et al. Exercise-induced pulmonary hypertension: physiological basis and methodological concerns. Am J Respir Crit Care Med 2013; 187: 576-583.

4 Lewis GD, Bossone E, Naeije R, et al. Pulmonary vascular hemodynamic response to exercise in cardiopulmonary diseases. Circulation 2013; 128: 1470-1479.

5 Herve P, Lau EM, Sitbon O, et al. Criteria for diagnosis of exercise pulmonary hypertension. Eur Respir J 2015; 46: 728-737.

6 Kovacs G, Avian A, Olschewski H. Proposed new definition of exercise pulmonary hypertension decreases false positive cases. Eur Respir J 2016; 47: 1270-1273.

7 Portillo K, Torralba Y, Blanco I, et al. Pulmonary hemodynamic profile in chronic obstructive pulmonary disease. Int J Chron Obstruct Pulmon Dis 2015; 10: 1313-1320.

8 Kovacs G, Avian A, Tscherner M, et al. Characterization of patients with borderline pulmonary arterial pressure. Chest 2014; 146: 1486-1493.

9 Lau EM, Chemla D, Godinas L, et al. Loss of vascular distensibility during exercise is an early hemodynamic marker of pulmonary vascular disease. Chest 2016; 149: 353-361.

10 Naeije R, Chesler N. Pulmonary circulation at exercise. In: Terjung R, ed. Comprehensive Physiology. Hoboken, John Wiley \& Sons, Inc., 2012.

11 Tolle JJ, Waxman AB, Van Horn TL, et al. Exercise-induced pulmonary arterial hypertension. Circulation 2008 118: 2183-2189.

12 Kafi SA, Mélot C, Vachiéry JL, et al. Partitioning of pulmonary vascular resistance in primary pulmonary hypertension. J Am Coll Cardiol 1998; 31: 1372-1376.

\section{Multilobar bilateral and unilateral chest radiograph involvement: implications for prognosis in hospitalised community- acquired pneumonia}

To the Editor:

Community-acquired pneumonia (CAP) remains a leading cause of morbidity and mortality worldwide [1]. Studies from the USA and Europe suggest that severe CAP patients requiring admission to the intensive care unit (ICU) reach a mortality of up to $39 \%[2,3]$.

Since potential poor prognosis is known to contribute to increased ICU admissions, anticipating complications through the use of supporting measurements becomes essential. The 2007 Infectious Diseases Society of America (IDSA)/American Thoracic Society (ATS) guidelines [1] have redefined severe CAP and indications for ICU admission, with a rule consisting of major and minor clinical criteria. The rule is considered positive if one major or three minor criteria are present [1]. The presence of multilobar infiltrates is included among the minor criteria. However, multilobar pneumonia can be bilateral or unilateral and this 